\title{
INFLUENCE OF MEDIUM COMPOSITION ON OXYGEN TRANSFER RATE IN ANIMAL CELL CULTURE
}

\author{
D. Toye, A. Galifi, T. Salmon, P. Marchot, E. Verdin, M. Crine \\ Laboratoire de Génie chimique, Université de Liège, \\ Institut de Chimie B6c - Sart Tilman , 4000 LIEGE, Belgium
}

\begin{abstract}
Experiments were conducted in a $0.25 \mathrm{~m}$ diameter bubble column to investigate the effect of animal cell culture medium composition on oxygen transfer rate. Air is used as the dispersed phase. The gas superficial velocity is varied between 0.4 and $2 \mathrm{~cm} / \mathrm{s}$ (aeration rate ranging between 0.05 and $0.25 \mathrm{vvm}$ ) and the bubble column is thus operated in the homogenous regime. Aqueous solutions the composition (electrolyte, protein concentrations) of which mimics a mammalian cell culture medium) are used as the continuous phase. In particular, the effect on oxygen transfer rate of additive such as PVP, Pluronic F-68 which are usually used to protect cells against local hydrodynamic stresses induced by bubble coalescence and bursting is addressed.

For each composition, the mass transfer coefficient is measured by the "gas in - gas out" method using a fast response dissolved oxygen probe. Its increase with gas velocity is measured. The liquid viscosity and surface tension are experimentally determined as a function of culture medium composition. Bubble size distribution is measured at different scales using three experimental techniques: gas disengagement technique, two dual optical probes and photography, which lead to results in good qualitative agreement one with each other. The integrated analysis of these data allows to decouple the effects of the different additive on coefficient $\mathrm{k}_{\mathrm{L}}$ and on the interfacial area, $a$.
\end{abstract}

Keywords: $\mathrm{k}_{\mathrm{L}} \mathrm{a}$, mass transfer, interfacial area, bubble size, bubble column, bioreactor, optical probe, disengagement technique, surfactant, antifoam

\section{INTRODUCTION}

Bubble columns are widely used as multiphase reactors in the chemical, petrochemical, pharmaceutical, food and environmental industries due to their intrinsic advantages, among which one may cite high heat and mass transfer rates, low operating and maintenance costs, absence of moving parts, simplicity of construction (Deckwer, 1992). They can be used as bioreactors for animal cells cultures (Zhang et al., 2004) in which cells are cultivated in suspension in the culture medium possibly fixed on microcarriers. Oxygen necessary for cell development is provided by bubbling of pure oxygen or oxygen enriched air at the column bottom.

One problem is that animal cells are known to be susceptible to damage potentially leading to cell death or reduced productivity, induced by hydrodynamic stress. In particular, they are very sensitive to the local hydrodynamic stress produced by the coalescence and the bursting of gas bubbles. Previous studies have shown that this effect affects mainly cells attached on bubble surface. It may be limited by the use of additives, such as Pluronic F68, which reduce interactions, and thus adherence, between cells and bubbles, or such as PVP (polyvinylpyrolidone) which increases bubble stability and thus reduces bubble bursting (Meier, 1999; Jordan, 1994).

The effects of additive come from modifications of liquid medium physico-chemical properties that can also have a significant influence on gas-liquid oxygen transfer rate, $\mathrm{k}_{\mathrm{L}} \mathrm{a}$. Previous studies have shown that an increase of liquid viscosity results in a decrease of $\mathrm{k}_{\mathrm{L}} \mathrm{a}$, due to an additional resistance to oxygen transfer. If liquid superficial tension is decreased, the effect on $\mathrm{k}_{\mathrm{L}}$ a is less obvious as the decrease of $k_{L}$ coefficient due to the additional resistance to transfer may be counterbalanced by an increase of the interfacial area, a, due to a smaller bubble diameter and/or to 
a higher gas holdup (Deckwer and Schumpe, 1993; Hikita et al., 1981). Culture media also contain, among others, electrolytes that inhibit coalescence and thus increase $\mathrm{k}_{\mathrm{L}}$ (Ribeiro and Mewes, 2007), proteins that may affect liquid phase viscosity and superficial tension and antifoam additive the effect of which on mass transfer has been reported in literature (Kawase and Moo-Young, 1990).

In order to get a better understanding of all these factors and their interactions, we have experimentally measured the effect of two additives, Pluronic F68 and PVP (34 kD and 58kD), on oxygen transfer rate, on bubble diameters and on gas holdup distribution in a bubble column filled with a synthetic culture medium containing electrolyte $(\mathrm{NaCl}$, $9 \mathrm{~g} / \mathrm{l}$ ) and protein (albumin, $100 \mathrm{mg} / \mathrm{l})$ ). The effect of two commercial antifoams has also been measured. The integrated analysis of experimental data allowed to decouple the effects of the different additive on coefficient $k_{L}$ and on the interfacial area, $a$.

\section{EXPERIMENTAL SETUP}

A $5 \mathrm{~m}$ high, $0.24 \mathrm{~m}$ internal diameter column filled with synthetic liquid culture medium, is fed from the bottom by compressed air through an industrial stainless steel multihole gas sparger (free area $0.35 \%, 203 \times 1 \mathrm{~mm}$ diameter holes or 4511 holes $/ \mathrm{m}^{2}$ ). Semi batch operation mode is adopted (fig.1). Air superficial velocity is varied between 0.4 and $2 \mathrm{~cm} / \mathrm{s}$, corresponding to aeration rates ranging between 0.05 and $0.25 \mathrm{vvm}$. The operating regime is homogenous.

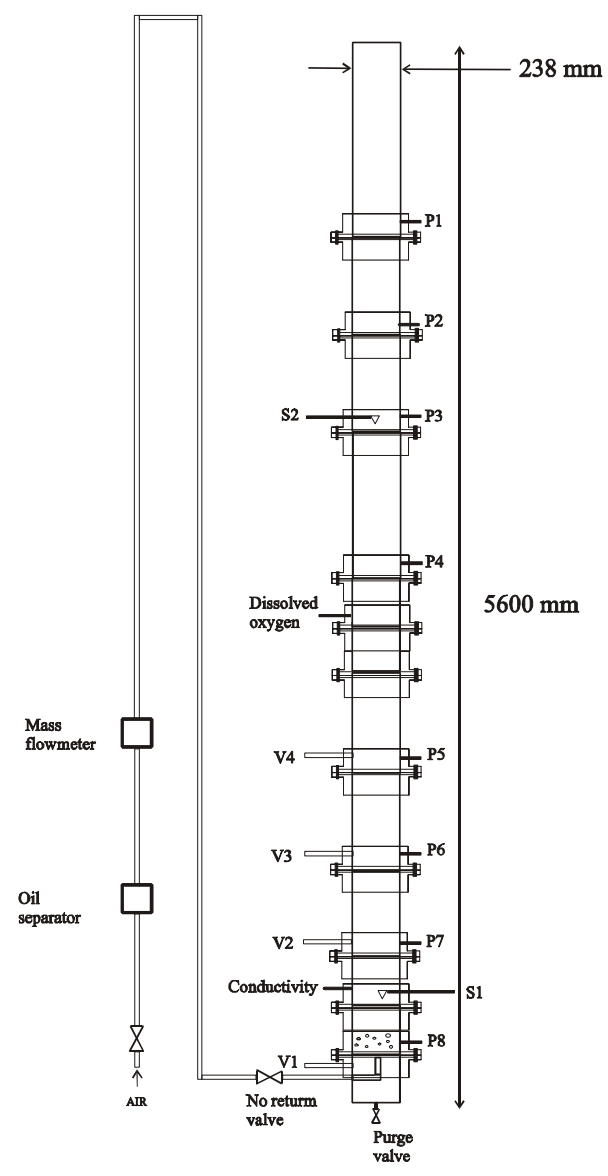

Fig. 1. Experimental bubble column. P1 - P8 : differential pressure probes. S1 - S2 : optical probes.

Two dual optical probes (RBI, France) with sapphire tips (S1 and S2) are used to measure local gas hold-up and bubble diameters. The lower probe is at $0.437 \mathrm{~m}$ from the sparger, the other $3.070 \mathrm{~m}$ higher. One of the column 
modules, situated approximately at mid-height, is made in Plexiglas and surrounded by a rectangular Plexiglas box filled with water, allowing to make pictures of the gas-liquid dispersion. Image analysis of these pictures gives access to bubble diameters in a small region situated near the column wall. Global value and axial profile of gas holdup are measured using eight non intrusive differential pressure probes P1 - P8 (Keller PR41 and PR25) are respectively located $4.530,4.043,3.507,2.758,1.756,1.257,0.751$ and $0.200 \mathrm{~m}$ above the sparger. P3 is also used to measured the distribution of gas bubble diameters using the gas disengagement technique, which is a method based on a bubble classification as a function of their upwards velocities (Patel et al., 1989).

A fast response dissolved oxygen probe (OxyGuard Ocean Probe - DO522M18) is used to measure oxygen transfer rate. The method used is the "gas in - gas out" method. The liquid phase is first completely deoxygenated using an inert gas (nitrogen). Column is then fed with air and the dissolved oxygen concentration increase is measured as a function of time. The probe response time, equal to $5.3 \mathrm{~s}$, is taken into account when $\mathrm{k}_{\mathrm{L}} \mathrm{a}$ is computed from oxygenation curves.

The liquid phase used is a synthetic culture medium containing electrolyte $(\mathrm{NaCl})$ and protein (albumin), the concentration of which, equal to $9 \mathrm{~g} / \mathrm{l}$ and $100 \mathrm{mg} / \mathrm{l}$, respectively, are similar to those encountered in real cell culture media. Two type of "cell protecting" additives have been tested: Pluronic F68, which is known to reduce cell attachment to bubble surface, and PVP which is known to increase bubble stability. Two types of PVP characterised by different molecular mass $(34 \mathrm{kD}$ and $58 \mathrm{kD}$ ) have been used. These additives were chosen because of they are highly soluble in aqueous medium, they are non toxic for cells and they are known to decrease liquid superficial tension. All additives were used in concentrations ranging between 0 and $0.15 \%$ in mass. The effect on oxygen transfer rate of two commercial antifoams, Sigma-Aldrich and Dow Corning Q7-2587, was also measured. The antifoam efficiency at the very small concentration used (50 ppm in mass), was checked in a standardized cell. In this cell, the height of foam formed during a given time ( 2 minutes) and the foam half-life after stopping gas injection may be measured.

Liquid medium viscosity was measured on a Broofield DV III - Ultra rheometer, equipped with a two concentric cylinder measurement cell. Liquid superficial tension was measured on a pendular drop tensiometer (Kruss DSA 100) equipped with a CCD camera and an image analysis software.

\section{RESULTS AND DISCUSSION}

\subsection{Liquid phase properties}

The effect of three cell protecting additives (Pluronic F68, PVP $34 \mathrm{kD}$ and PVP $58 \mathrm{kD}$ ) on viscosity and surface tension of the synthetic culture medium containing $100 \mathrm{mg} / \mathrm{l}$ of albumin and $9 \mathrm{~g} / \mathrm{l}$ of $\mathrm{NaCl}$ have been measured as a function of additive concentration. Fig. $2 \mathrm{a}$ and $2 \mathrm{~b}$ show that the viscosity and the surface tension of culture medium with no additive (concentration equal to 0 ) are approximately the same as for water at the same temperature, equal to $83 \mathrm{cPo}$ for viscosity and to $73.4 \mathrm{cPo}$ for surface tension. At the concentrations tested, albumin and $\mathrm{NaCl}$ thus don't influence liquid phase properties.

Fig.2a that presents the influence of additive on viscosity shows that both types of PVP (34 kD and $58 \mathrm{kD})$ have no influence on liquid phase viscosity, whereas an relative increase of medium viscosity of $70 \%$ is observed when Pluronic F68 is increased from 0 to $0.15 \%$. Fig.2b presents surface tension results. It shows that, even used at very low concentration, all additives act as surfactants as the liquid medium surface tension is significantly reduced. The most important influence is observed for high molecular weight PVP $(58 \mathrm{kD})$ : a reduction of $7 \%$ is measured at a mass concentration of $0.15 \%$.

From these results, one may expect that PVP $(34 \mathrm{kD}$ and $58 \mathrm{kD})$ has a less unfavourable effect than Pluronic F68 on oxygen transfer rate as PVP does not introduce any additional resistance due to increased viscosity and its surfactant effect is more pronounced. 
(a)

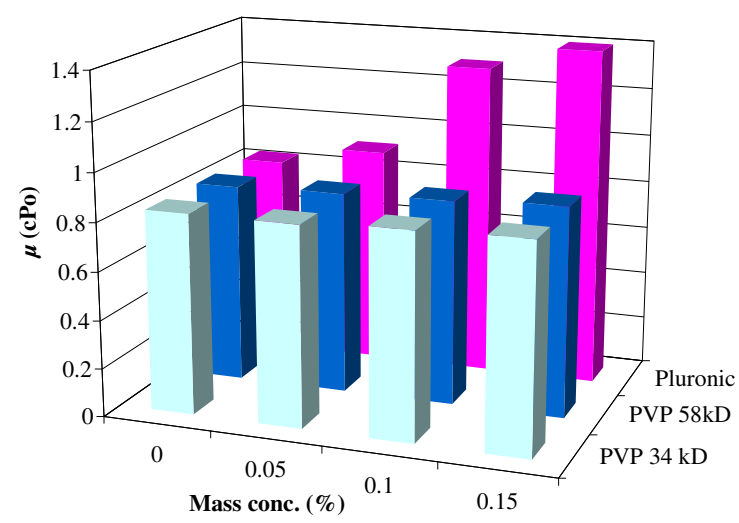

PVP 34 kD $\square$ PVP 58kD — Pluronic F68 (b)

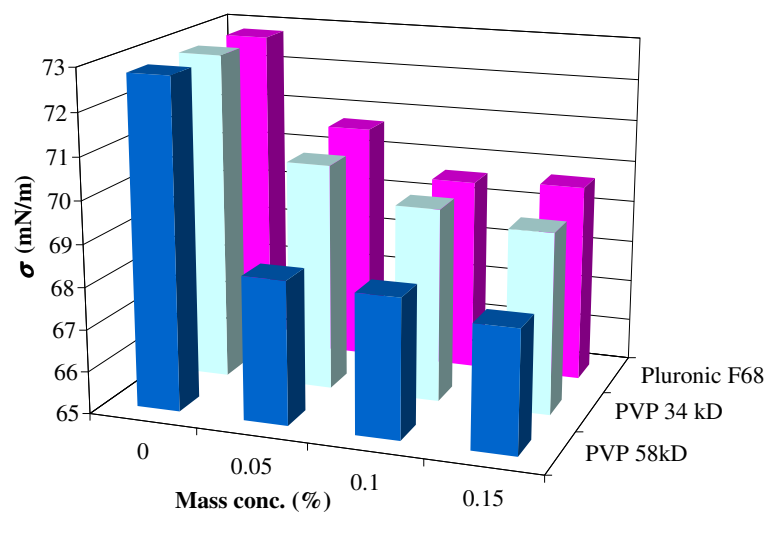

PVP 58kD PVP 34 kD — Pluronic F68

Fig. 2 : Effect of cell protecting additives on liquid medium properties : (a) viscosity (b) surface tension

\subsection{Gas phase properties}

In order to determine the effect of the tested additives on the gas-liquid interfacial surface area, quantities related to the gas phase distribution in the bubble column have been measured. Evolution of gas holdup as a function of gas velocity was measured for different additive concentrations. In all cases, the mean bubble size was measured at two different heights in the column, which allows detecting a possible effect on the coalescence phenomenon.

\section{Gas holdup:}

Curves on Fig.3a correspond to global gas holdup values measured between pressure probes P1 and P8 as a function of gas velocity. Cell protecting additives were used at a concentration equal to $0.1 \%$ in mass to determine their influence. In all cases, gas holdup increases linearly with gas superficial velocity, which confirms that column operates in the bubble flow regime. All additives lead to an increase of gas holdup. The effect of Pluronic F68 and PVP $34 \mathrm{kD}$ is similar and not very important (less than 10\% relative). On the contrary, the influence of PVP $58 \mathrm{kD}$ is more pronounced, as one observe an relative increase approximately equal to $50 \%$. These results are in good agreement with the surfactant effects reported in the preceding section.

(a)

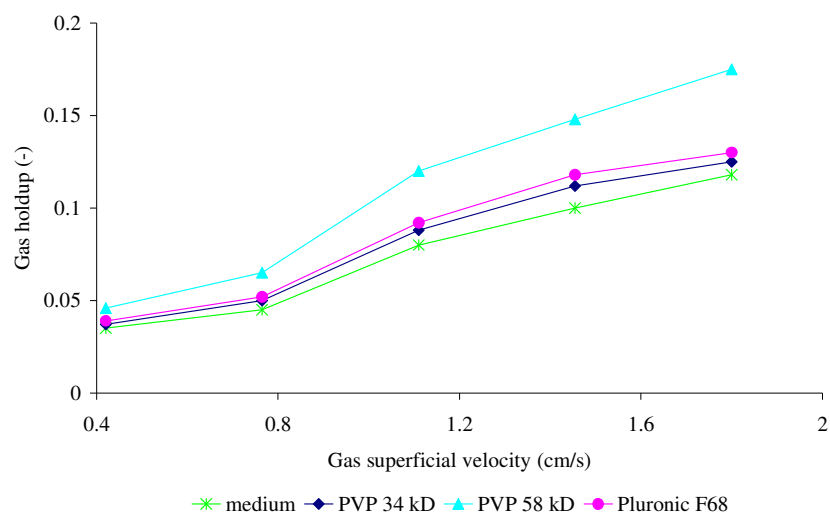

(b)

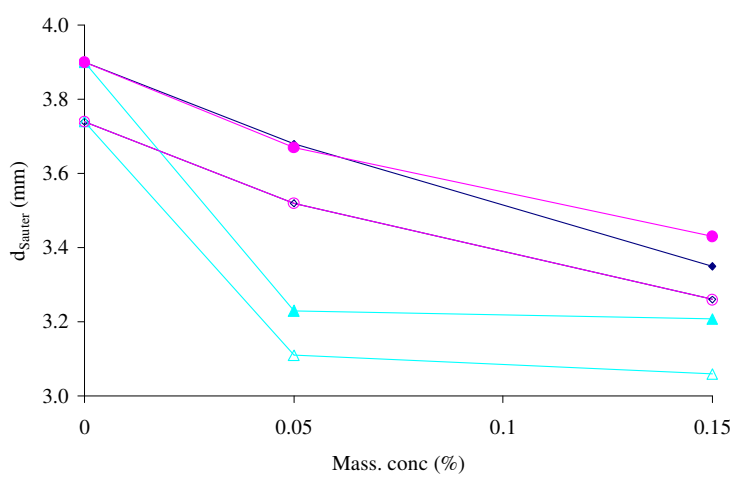

$\rightarrow$ PVP $34-$ PVP $34 \triangle$ PVP $58 \rightarrow$ PVP $58-$ Pluronic $\rightarrow$ Pluronic

Fig. 3: Influence of cell protecting additive on gas phase distribution (a) Global gas holdup vs gas superficial velocity for additive concentrations equal to $0.1 \%$ in mass - (b) Bubble Sauter diameter measured by optical probes located $0.437 \mathrm{~m}$ (empty symbols) and 3.507 (full symbols) from the sparger for a gas velocity equal to $0.42 \mathrm{~cm} / \mathrm{s}$.

\section{Bubble diameter}

Bubble diameter was measured independently with three experimental techniques: optical probes, photography and gas disengagement. Results obtained with these techniques are in good qualitative agreement (Galifi, 2008), that's the reason why we shall only present here diameter values measured with optical probes. Results are presented on 
Fig. 3b. They correspond to measurements performed at two different heights in the column (S1 and S2 on Fig 1) for a gas superficial velocity equal to $0.42 \mathrm{~cm} / \mathrm{s}$ and two additive concentrations $(0.05$ and $0.15 \%$ in mass). All additives lead to a decrease of bubble diameter. Influences of Pluronic F68 and PVP 34kD are similar, whereas and the effect of PVP $58 \mathrm{kD}$ is more pronounced. These results are in perfect agreement with the surfactant effects reported in the preceding section.

Results on Fig 3b show that, even in absence of additive (concentration equal to zero), no significant coalescence phenomenon may be observed in the liquid medium. In all cases, a relative increase of bubble diameter of 5\% in the mean is observed between the lower probe (empty symbols) and the higher one (full symbols). It is smaller than the diameter increase that can be attributed to gas dilation with hydrostatic pressure drop along bubble upwards travel in the column $(3.07 \mathrm{~m}$ liquid $=30.1 \mathrm{kPa})$. If air is assumed to be a perfect gas, this pressure drop corresponds to dilation of $22 \%$. If bubbles are assumed to be spherical in shape, it corresponds to a diameter increase of $7 \%$. This observation is confirmed by gas holdup vertical profiles measured by eight differential pressure probes (P1 to P8) distributed along the column wall. These profiles, not shown here due to lack of place, show that in all tested conditions, holdup increases linearly when going up in the column.

Results relative to gas phase distribution thus show that all cell protecting additives should all have a favourable effect on gas-liquid interfacial surface area, as they lead to an increase of gas holdup along with a decrease of bubble diameter. The most pronounced influence is observed for PVP $58 \mathrm{kD}$, that is thus likely to be the best candidate.

\subsection{Oxygen transfer rate}

The oxygen transfer rate is classically quantified by the value of $\mathrm{k}_{\mathrm{L}} \mathrm{a}$, which is the product of a mass transfer coefficient $\mathrm{k}_{\mathrm{L}}$ and of the gas-liquid interfacial area, a. $\mathrm{k}_{\mathrm{L}}$ a values were measured as a function of gas velocity by the gas in gas out method using a fast oxygen probe. Fig. 4a shows the influence on $\mathrm{k}_{\mathrm{L}} \mathrm{a}$ of additives used at a concentration equal to $0.1 \%$ in mass: values measured with additives are compared to values measured without additive (culture medium). Curves on Fig. 4a show that $\mathrm{k}_{\mathrm{L}} \mathrm{a}$ increases almost linearly with gas velocity. Pluronic F68 leads to a decrease of $\mathrm{k}_{\mathrm{L}}$ a. The surfactant effect of Pluronic F68 and its positive influence on gas distribution is thus not high enough to counterbalance the resistance to transfer increase due to the higher viscosity. $\mathrm{k}_{\mathrm{L}} \mathrm{a}$ values measured with PVP $(34 \mathrm{kD}$ and $58 \mathrm{kD})$ are increased, the last one having a more pronounced effect than the former, which can be explained by its more important surfactant effect.

Fig $4 \mathrm{~b}$ presents $\mathrm{k}_{\mathrm{L}}$ a values measured at $0.42 \mathrm{~cm} / \mathrm{s}$ for different additive concentrations. It shows that the positive or negative influence of each additive varies in a logical way with concentration.
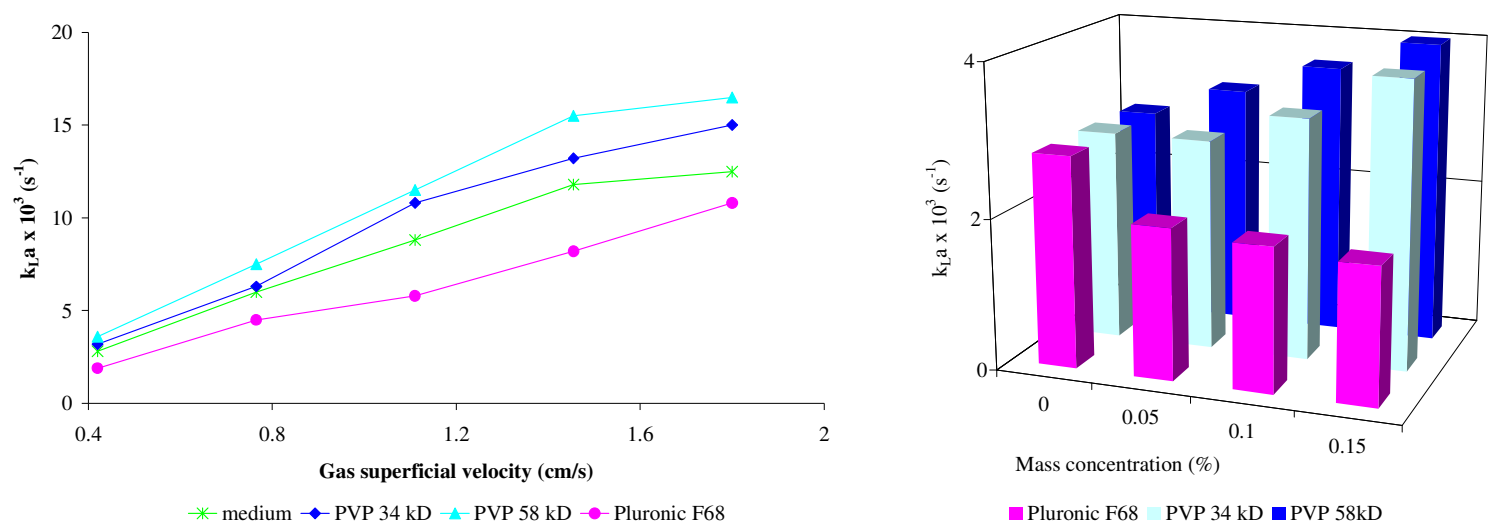

Fig.4: Influence of cell protecting additives on $\mathrm{k}_{\mathrm{L}} \mathrm{a}$ (a) Evolution with gas velocity (additive conc. $=0.1 \%$ mass) (b) Influence of additive concentration (air superficial velocity $=0.42 \mathrm{~cm} / \mathrm{s}$ ).

The $\mathrm{k}_{\mathrm{L}}$ a values reported here may seem very low by comparison with values reported in the literature (Hikita, 1981). They can be explained by the small air velocities used, that correspond to aeration conditions used in animal cell cultures $(0.05-0.25 \mathrm{vvm})$. 


\subsection{Antifoam}

The antifoams used (Sigma and DC) have no significant effect on the oxygen transfer rate, which can be explained by the absence of effect on liquid (viscosity and surface tension) and gas properties (gas holdup, bubble diameter). Efficiency tests performed in a standardized cell have shown that in every case, the height of formed foam and the foam half-life are reduced by a factor two, approximately. Antifoam Sigma is more convenient to use because it is highly soluble in aqueous liquid solutions, on the contrary to antifoam DC (non dissolved solid particles remain in suspension).

\section{CONCLUSIONS}

In this experimental work, the influence of three cell protecting additives on quantities that may affect the oxygen transfer rate was measured. Qualitative results relative to liquid viscosity and surface tension, to gas holdup and bubble diameter and to $\mathrm{k}_{\mathrm{L}}$ a values are presented in Table 1. It shows that the effect on oxygen transfer rate which depends on the additive may be related to other measured quantities. Pluronic F68 leads to a significant decrease of $\mathrm{k}_{\mathrm{L}} \mathrm{a}$, whereas $\mathrm{k}_{\mathrm{L}}$ a values measured with PVP $(34 \mathrm{kD}$ and $58 \mathrm{kD})$ are higher, the last one having a more pronounced effect than the former. An integrated analysis of all the data allows decoupling the effects on coefficient $k_{L}$ and on the interfacial area, $a$, and confirms the strong influence of liquid viscosity and surface tension. A decrease of $\mathrm{k}_{\mathrm{L}}$ due to a higher viscosity value seems to be difficult to counterbalance by an increase of interfacial area due to surfactant effect as shown in the case of Pluronic F68. For additives that have no significant influence on viscosity, such as PVP $34 \mathrm{kD}$ and PVP $58 \mathrm{kD}$, an increase of surface tension leads to higher interfacial area and thus to higher $\mathrm{k}_{\mathrm{L}} \mathrm{a}$ values. The oxygen transfer rate is thus improved.

Table 1 Effect of additives used at a concentration of $0.15 \%$ in mass

(- for decrease; + for increase; = for no effect)

\begin{tabular}{cccccc} 
Additive & Viscosity & $\begin{array}{c}\text { Surface } \\
\text { tension }\end{array}$ & Gas holdup & $\begin{array}{c}\text { Bubble } \\
\text { diameter }\end{array}$ & $\mathrm{k}_{\mathrm{L}} \mathrm{a}$ \\
\hline PVP 35 kD & $=$ & - & + & + & + \\
PVP 58 kD & $=$ & -- & ++ & ++ & ++ \\
Pluronic F68 & + & - & + & + & -
\end{tabular}

Before drawing definite conclusions, one has to keep in mind that no coalescence phenomenon was observed in the present study, certainly due to the very low gas velocities used. More experiments should be realised in a larger range of operating conditions possibly leading to coalescence phenomena, to confirm the influence of liquid surface tension on oxygen transfer rate.

\section{REFERENCES}

Deckwer, W.D. (1992). Bubble column reactors, Wiley, Chichester.

Deckwer, W.D. and Schumpe, A. (1993) Improved tools for bubble column reactor design and scale-up, Chemical Engineering Science, 48, 889-911.

Galifi A. (2008), Étude de l'aération dans un milieu de culture cellulaire, MSc Thesis, Université de Liège, Liège, Belgium.

Hikita, H., Asai, S., Tanigawa, K., Segawa, K., Kiato, M. (1981) The volumetric mass transfer coefficient in bubble columns, Chemical Engineering Journal, 22, 61-67.

Jordan, M. (1994) Interactions between animal cells and gas bubbles: the influence of serum and Pluronic F68 on the physical properties of bubble surface, Biotechnology and Bioengineering, 43, 448-454.

Kawase Y and Moo-young, M. (1990) The effect of antifoam on mass transfer in bioreactors, Bioprocess and Bioengineering, 169, 43-47.

Meier, S.J. (1999), Cell death from bursting bubbles: role of cell attachment to rinsind bubble in sparged bioreactor, Biotechnology and Bioengineering, 62, 468-478.

Patel, S.A., Daly, J.G and Bukur, D.B. (1989) Holdup and interfacial measurements usind dynamic disengagement, AIChE Journal, 35, 931-942.

Ribeiro, C.P. and Mewes, D. (2007) The influence of electrolytes on gas hold-up and regime transition in bubble columns, Chemical Engineering Science, 62, 4501-4509.

Zhang, S., Handa-Corrigan, A., Spier, R.E. (2004) Oxygen transfer properties of bubbles in animal cell culture media, Biotechnology and Bioengineering, 40, 252-259. 